\title{
Stellar perturbations on the scattered disk
}

\author{
H. Rickman ${ }^{1}$, Ch. Froeschlé ${ }^{2}$, Cl. Froeschlé ${ }^{2}$, and G. B. Valsecchi ${ }^{3}$ \\ 1 Uppsala Astronomical Observatory, Box 515, 75120 Uppsala, Sweden \\ e-mail: hans.rickman@astro.uu.se \\ 2 Observatoire de la Côte d'Azur, BP 4229, 06304 Nice, France \\ 3 INAF-IASF, via Fosso del Cavaliere 100, 00133 Roma, Italy \\ e-mail: giovanni@rm.iasf.cnr.it
}

Received 16 April 2004 / Accepted 25 August 2004

\begin{abstract}
We present a statistical model for estimating the effects of stellar encounters on orbits in the outer Solar System, focussing on the scattered disk at $\lesssim 10^{3}$ AU from the Sun. We describe a Monte Carlo simulation using those results and apply it to the evolution of the scattered disk over $4 \mathrm{Gyr}$, finding that a final perihelion distance distribution with an extended tail reaching to very large values is to be expected. This would likely result from a single close stellar encounter, in agreement with the conclusion by Morbidelli \& Levison (2004). We estimate that the newly discovered minor planet (90377) Sedna may be a typical representative of such an extended scattered disk and that a few more objects of the same size may reside at similar heliocentric distances. There is a possibility that the bulk of the population, which should have smaller perihelion distances, contains some very large objects that may have contributed to sculpting the Kuiper Belt. We also find that the creation of an extended scattered disk by a stellar encounter should have been accompanied by a huge influx of large objects into the inner Solar System, but the timing of the encounter is constrained by the fact that the scattered disk must still have been quite massive. Thus it likely happened long before the purported late heavy bombardment of the terrestrial planets.
\end{abstract}

Key words. comets: general - Kuiper Belt - minor planets, asteroids

\section{Introduction}

The discovery of minor planet (90377) Sedna was recently reported (Brown et al. 2004). This is a very large object, whose absolute magnitude $H=1.7$ and limits to its thermal radiation indicate a diameter intermediate between (50000) Quaoar and Pluto. Orbital elements determined on the basis of observations from Sep. 2001 until early 2004 include a perihelion distance of $q \simeq 76 \mathrm{AU}$ and a semimajor axis of $a \simeq 532 \mathrm{AU}$ (Marsden 2004), and the object is currently at $r \simeq 90$ AU inbound.

Both the size and the orbit of Sedna are remarkable and came quite unexpected. The closest analogue among previous transneptunian objects is $2000 \mathrm{CR}_{105}$ with $q \simeq 44 \mathrm{AU}$ and $a \simeq 230 \mathrm{AU}$, whose $H=6.1$ indicates a diameter around $300 \mathrm{~km}$. Both these objects belong to the "extended scattered disk" according to the terminology of Gladman et al. (2002), as Morbidelli \& Levison (2004) note in their analysis of formation scenarios. The orbit of Sedna is of special importance in that it accentuates very clearly the need for an efficient mechanism to raise the perihelion distances from the range accessible by interactions with Neptune (Gladman et al. 2002; Gomes 2003); at variance with other trans-Neptunian objects, Sedna is really the propotype of what we will call, in the rest of this paper, the ETNOs, the Exterior Trans-Neptunian Objects.
Let us briefly recapitulate some current ideas about the scattered disk. It is a structure that arises naturally from the accretion of the giant planets. Gravitational scattering sends a large population of planetesimals and planetary embryos both inward (toward the Sun) and outward to orbits with larger semimajor axes. The latter have perihelia locked to the vicinity of the scattering planet's orbit, and aphelia reaching out to hundreds or thousands of AU. The orbital inclinations are small to moderate. At the time the planetary accretion reaches and passes its maximum rate, the mass of this exterior disk may be comparable to the final mass of the planet. Afterwards it gradually subsides, mainly through repeated encounters with the planet, which either eject the objects into hyperbolic orbits or increase their semimajor axes beyond the range where external perturbers take control of the perihelion distances. Today's scattered disk - even though perhaps comparable in population size to the Kuiper Belt (Morbidelli \& Brown 2004) - is hence but a bleak shadow of what it was, when the giant planets accreted.

Brown et al. (2004) discussed modes of origin for Sedna, concentrating on three mechanisms - scattering by an unseen planet at $r \sim 70 \mathrm{AU}$, a single stellar encounter, and formation of the Solar System in a stellar cluster. They did not exclude any of these scenarios, but found a preference for the third one. The main argument against the unseen planet was 
that in fact it should likely have been seen, while the stellar encounter was deemed too unlikely based on statistical results by Fernández (1997). The stellar cluster scenario was found credible on the basis of an investigation by Fernández \& Brunini (2000). Let us note, however, that this scenario may suffer from a time scale problem. Galactic clusters tend to disperse within $\lesssim 10^{8} \mathrm{yr}$, as evidenced by the main sequence turnoff points of most observed clusters, and it is not certain that the nascent Solar System could form a scattered disk exterior to Neptune's orbit and extending to $10^{3} \mathrm{AU}$ within such a short time interval. For a relevant set of preliminary results, see Dones et al. (2004).

Morbidelli \& Levison (2004) considered an early high eccentricity of Neptune's orbit, massive planetary embryos in the transneptunian region, tidal effects of an early, massive transneptunian disk, and stellar encounters. Their conclusion, based on actual simulations, was that only the stellar encounter scenario gave satisfactory results. An important finding was that too close an encounter, at 500 AU or less, would yield too many objects with semimajor axes much less than the two discovered objects (2000 $\mathrm{CR}_{105}$ and Sedna) in the extended scattered disk. Their preferred distance was $800 \mathrm{AU}$. The stellar velocity employed in their simulation $\left(0.2 \mathrm{AU} / \mathrm{yr} \sim 1 \mathrm{~km} \mathrm{~s}^{-1}\right)$ is conspiciously small compared to the distribution of Galactic field stars in the solar neighbourhood (cf. Table 1) - instead it is suggestive of the velocity that a member of the Sun's hypothetical stellar cluster would have.

Thus, except if viewed in the stellar cluster scenario, the very puffed-up $q$ distribution resulting from the Morbidelli \& Levison simulation (their Fig. 5c) may not be realistic. We are currently performing Monte Carlo simulations of stellar perturbations on comets of the Oort cloud, aiming to use a distribution of stellar parameters (velocities, masses, relative encounter frequencies) that is as realistic as possible. Upon the discovery of Sedna, by the time of the submission of the above-cited papers by Brown et al., and Morbidelli \& Levison, we decided to check the apparently promising stellar encounter scenario on the basis of our own simulation procedure. The model developed for this case is a slight modification of the one we use for the Oort cloud and focuses on a scattered disk component with perihelia slightly outside Neptune's orbit and aphelia extending to $10^{3} \mathrm{AU}$ - we refer to such hypothetical objects as "proto-ETNOs".

In Sect. 2 we describe this model, in Sect. 3 we present the results, and in Sect. 4 we discuss their relevance and implications for the structure of the extended scattered disk, judging that indeed a stellar encounter is a likely scenario without excluding the possibility of a stellar cluster. Speculations about effects on the classical Kuiper belt and a discussion relating to the late heavy bombardment are also offered. Finally, Sect. 5 summarizes our conclusions.

\section{Modelling of stellar perturbations}

We simulate stellar perturbations in the following manner. First we create impulse samples for objects placed at certain standard heliocentric distances $r_{\mathrm{s}}$. In the present investigation we use $r_{\mathrm{s}}=100,200,500$ and 1000 AU. Each sample involves $1 \times 10^{7}$ stellar passages within $40000 \mathrm{AU}$ from the Sun, assumed to occur at random with an isotropic distribution of distance and direction with respect to the position of the object. For convenience the calculations are done with the star passing in the $z$ direction of a cartesian coordinate system centered on the Sun such that the star cuts the $(x, y)$ plane on the positive $x$ axis, while the object is placed at a random position on a sphere of radius $r_{\mathrm{s}}$ around the Sun.

We use the classical impulse approximation, i.e., the star moves along a straight line with constant speed $v_{*}$, and the object remains fixed. The heliocentric impulse imparted to the object, i.e., the differential impulse of the object with respect to the Sun, is hence:

$\Delta \boldsymbol{v}=\frac{2 G M_{*}}{v_{*}}\left\{\frac{\boldsymbol{d}}{d^{2}}-\frac{\boldsymbol{d}_{\odot}}{d_{\odot}^{2}}\right\}$

where $G$ is the gravitational constant, $M_{*}$ is the mass of the star, and $\boldsymbol{d}$ and $\boldsymbol{d}_{\odot}$ are the vectors from the object and the Sun, respectively, to the closest points on the stellar track. We count distances in AU, time in years and masses in solar masses, and thus: $G=4 \pi^{2}$. We have:

$$
\left.\begin{array}{l}
\boldsymbol{d}=\left(d_{\odot}-x,-y, 0\right) \\
\boldsymbol{d}_{\odot}=\left(d_{\odot}, 0,0\right)
\end{array}\right\}
$$

where $\boldsymbol{r}=(x, y, z)$ is the heliocentric position vector of the object and thus:

$x^{2}+y^{2}+z^{2}=r_{\mathrm{s}}^{2}$.

For each stellar passage we pick a random number $\xi$ uniformly between 0 and 1 (henceforth, the symbol $\xi$ will always denote a new random number with this distribution), and we calculate $d_{\odot}=40000 \sqrt{\xi}$. For symmetry we restrict our attention to positive values of $z$, and we take $z=\xi r_{\mathrm{s}}$. We then compute $\alpha=2 \pi \xi$, and $x=\sqrt{r_{\mathrm{s}}^{2}-z^{2}} \cos \alpha$ and $y=\sqrt{r_{\mathrm{s}}^{2}-z^{2}} \sin \alpha$. The impulse vector is then computed from Eq. (1) using a random choice of $M_{*}$ and $v_{*}$, as follows.

We consider the same 13 categories of stars as in García-Sánchez et al. (2001) with the values of solar apex velocities, internal velocity dispersions and encounter frequencies listed by these authors (see their Table 8) as derived from classical stellar statistics investigations. For the stellar masses we take values listed by Allen (1985). In Table 1 we list all stellar parameters used in our work. To calculate the encounter speed of a certain star, we first determine the category from the fractional contributions to the total encounter frequency of 10.525 $\mathrm{Myr}^{-1}$ within $d_{\odot}<1 \mathrm{pc}$.

We compute the peculiar velocity $\boldsymbol{u}_{*}$ of the star with respect to the velocity centroid of its category, such that $u_{*}=\left|\boldsymbol{u}_{*}\right|$ is found from a combination of three Gaussian components, each with the dispersion listed in Table 1 . We then combine this with the apex velocity $\boldsymbol{v}_{\odot}$ assuming a random direction of $\boldsymbol{u}_{*}$ :

$v_{*}=\left\{v_{\odot}^{2}+u_{*}^{2}-2 v_{\odot} u_{*} \cos \beta\right\}^{1 / 2}$

with $\cos \beta$ picked at random according to $\cos \beta=2 \xi-1$.

The four impulse samples are used as input to a Monte Carlo simulation of stellar encounters for orbits like that of a "proto-ETNO". To this end we first determine a minimum impulse modulus $|\Delta v|_{\min }$ such that only impulses larger than this 
Table 1. Stellar parameters. Categories 1-11 refer to MK types for main sequence stars, category 12 is white dwarfs, and category 13 is giant stars. Values of the solar apex velocities $v_{\odot}$ and encounter frequencies $f_{\text {enc }}$ (the latter in number per Myr within 1 pc) have been taken from Table 8 of García-Sánchez et al. (2001), while the velocity dispersions $\sigma_{*}$ are one-dimensional and correspond to those listed by García-Sánchez et al. divided by $\sqrt{3}$. Stellar masses are taken from Allen (1985).

\begin{tabular}{cc|cccc}
\hline \hline Stellar type & $N$ & $\begin{array}{c}v_{\odot} \\
\left(\mathrm{km} \mathrm{s}^{-1}\right)\end{array}$ & $\begin{array}{c}\sigma_{*} \\
\left(\mathrm{~km} \mathrm{~s}^{-1}\right)\end{array}$ & $f_{\text {enc }}$ & $\begin{array}{c}\text { Mass } \\
\left(M_{\odot}\right)\end{array}$ \\
\hline B0 & 1 & 18.6 & 8.5 & 0.005 & 18 \\
A0 & 2 & 17.1 & 11.4 & 0.03 & 3.2 \\
A5 & 3 & 13.7 & 13.7 & 0.04 & 2.1 \\
F0 & 4 & 17.1 & 16.8 & 0.15 & 1.7 \\
F5 & 5 & 17.1 & 20.9 & 0.08 & 1.3 \\
G0 & 6 & 26.4 & 21.6 & 0.22 & 1.1 \\
G5 & 7 & 23.9 & 22.6 & 0.35 & 0.93 \\
K0 & 8 & 19.8 & 19.7 & 0.34 & 0.78 \\
K5 & 9 & 25.0 & 25.1 & 0.85 & 0.69 \\
M0 & 10 & 17.3 & 24.7 & 1.29 & 0.47 \\
M5 & 11 & 23.3 & 24.1 & 6.39 & 0.21 \\
wd & 12 & 38.3 & 36.6 & 0.72 & 0.9 \\
gi & 13 & 21.0 & 23.7 & 0.06 & 4 \\
\hline
\end{tabular}

are taken into account. This determination is described below, and the aim is to find a balance such that $|\Delta v|<|\Delta v|_{\text {min }}$ can safely be disregarded, and encounters with $|\Delta \boldsymbol{v}|>|\Delta \boldsymbol{v}|_{\min }$ are in general close enough for the classical impulse approximation to be valid. Thus, for each perturbation sample we only save the entries with $|\Delta v|>|\Delta v|_{\min }$, and we note the measure of this subsample compared to the total sample size of $1 \times 10^{7}$. We could have reached a somewhat higher accuracy of our simulation results, if we had used more than four impulse samples. However, as illustrated by Table 2 below, the four samples are not dramatically different, and we judge this loss of accuracy to be no more severe than that caused by using the classical impulse approximation.

Each test object in our simulation is initially placed on an orbit with perihelion distance $q_{0}=35 \mathrm{AU}$ and aphelion distance $Q_{0}=1000 \mathrm{AU}$ at a time $4 \mathrm{Gyr}$ ago. By this we aim to represent a scattered disk object that was ejected by Neptune and had its perihelion distance raised until it almost decoupled from the planet's gravitational influence. The simulation runs for 4 Gyr with a time step $\Delta t=2.525 \times 10^{4} \mathrm{yr}$. This is exactly $1 \%$ of the average interval between consecutive stellar passages within $40000 \mathrm{AU}$ for the above choice of the encounter frequency, i.e., the probability for a random encounter to occur during time $\Delta t$ is $1 \%$. For each time step we decide by means of a random number $\xi$ if a stellar passage occurs or not.

If a passage occurs, we calculate the exact time $t \in\left[t_{0}, t_{0}+\right.$ $\Delta t$ ] by a random number $\xi$, i.e., $t=t_{0}+\xi \Delta t$, and we solve Kepler's equation to find the eccentric anomaly and the heliocentric distance $r$ of the object. We then associate the encounter with one of the four standard distances $r_{\mathrm{s}}$ according to limits placed at the geometric means of consecutive $r_{\mathrm{s}}$ values: if $r<141.4$ we take $r_{\mathrm{s}}=100$, else if $r<316.2$ we take $r_{\mathrm{s}}=200$, else if $r<707.1$ we take $r_{\mathrm{s}}=500$, and otherwise we take $r_{\mathrm{s}}=1000$. With the above combination of $q_{0}$ and $Q_{0}$ we get likelihoods of $3.0 \%, 6.3 \%, 26.2 \%$ and $64.5 \%$ for the four representative values of $r_{\mathrm{s}}$.

We first check by means of a random number $\xi$ if the encounter yields a significant impulse, and if it does, we pick a random entry from the corresponding file. These entries contain both $|\Delta v|$ and the direction cosine $C_{r}=\widehat{\Delta \boldsymbol{v}} \cdot \widehat{\boldsymbol{r}}$ with respect to the radial direction (the hats denote unit vectors). In addition we pick at random the direction cosine $C_{t}$ of the non-radial component with respect to the transverse direction in the orbital plane. We can hence compute the perturbations $\Delta v_{r}$ and $\Delta v_{t}$ of both in-plane velocity components as well as the out-of-plane component $\Delta v_{n}$.

By applying all these we recompute the orbital energy and angular momentum, and thus, we get the new perihelion distance $q$ and inverse semimajor axis $1 / a$. We take another time step $\Delta t$ and repeat the whole process, until the total time elapsed reaches $4 \times 10^{9} \mathrm{yr}$.

As a supplement to this investigation we have also developed a routine to compute the effect of a single close stellar encounter on a population of objects like the scattered disk. We still use the classical impulse approximation, and we let the star travel parallel to the $z$-axis as in the above-described calculation of the impulse samples. The disk is formed by a large number of test objects with uniform distributions of $a$ and $q$ within prechosen limits. The orbits have inclinations to the midplane that are confined below a given maximum value, and the orbital position of a given object is chosen at random, uniformly in mean anomaly. The perturbations of $q$ and $a$ for all the disk objects are computed and recorded. Finally, the orientation of the disk can be chosen such that the axis of symmetry is either the $x, y$ or $z$ coordinate axis. This means that the star can cross the disk perpendicular to its plane (the $z$-disk) or along a chord in the midplane (the $y$-disk), or it may pass close to the disk parallel to its plane without crossing it (the $x$-disk).

\section{Results}

Let us first check the validity of the classical impulse approximation for our study. In fact, the reliability of the simulation results is not critically dependent on a high accuracy of the estimates of $\Delta \boldsymbol{v}$. It should be enough to check against total breakdown of the underlying assumptions. To this end we compute the time spent by the star along the inner part of its track, where most of the interaction occurs, and compare it with the orbital period of a typical "proto-ETNO" - the latter being a little more than $10000 \mathrm{yr}$.

We compute the interaction time as: $T_{*}=D_{*} / v_{*}$, where the interaction length $D_{*}$ is estimated by

$D_{*}=\max \left(2 \sqrt{3} d_{\odot}, 5000 \mathrm{AU}\right)$

i.e., either the distance between the two points where the heliocentric distance equals $2 d_{\odot}$, or $5000 \mathrm{AU}$, whichever is larger (see Fig. 1). This definition guarantees that indeed most of the interaction occurs within the segment of length $D_{*}$, independent of the encounter geometry.

Figure 2 shows histograms of $T_{*}$ for encounter subsamples with $|\Delta v|_{\text {min }}=0.1 v_{\text {ref }}$, where the reference speed is arbitrarily 


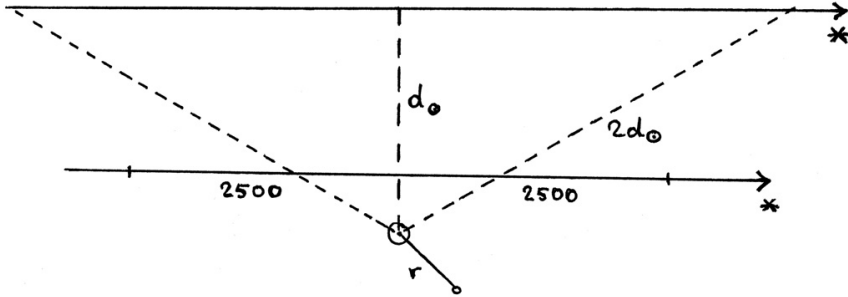

Fig. 1. Encounter geometry and illustration of the interaction length. For the upper stellar track the latter is $2 \sqrt{3} d_{\odot}$, and for the lower it is $5000 \mathrm{AU}$.
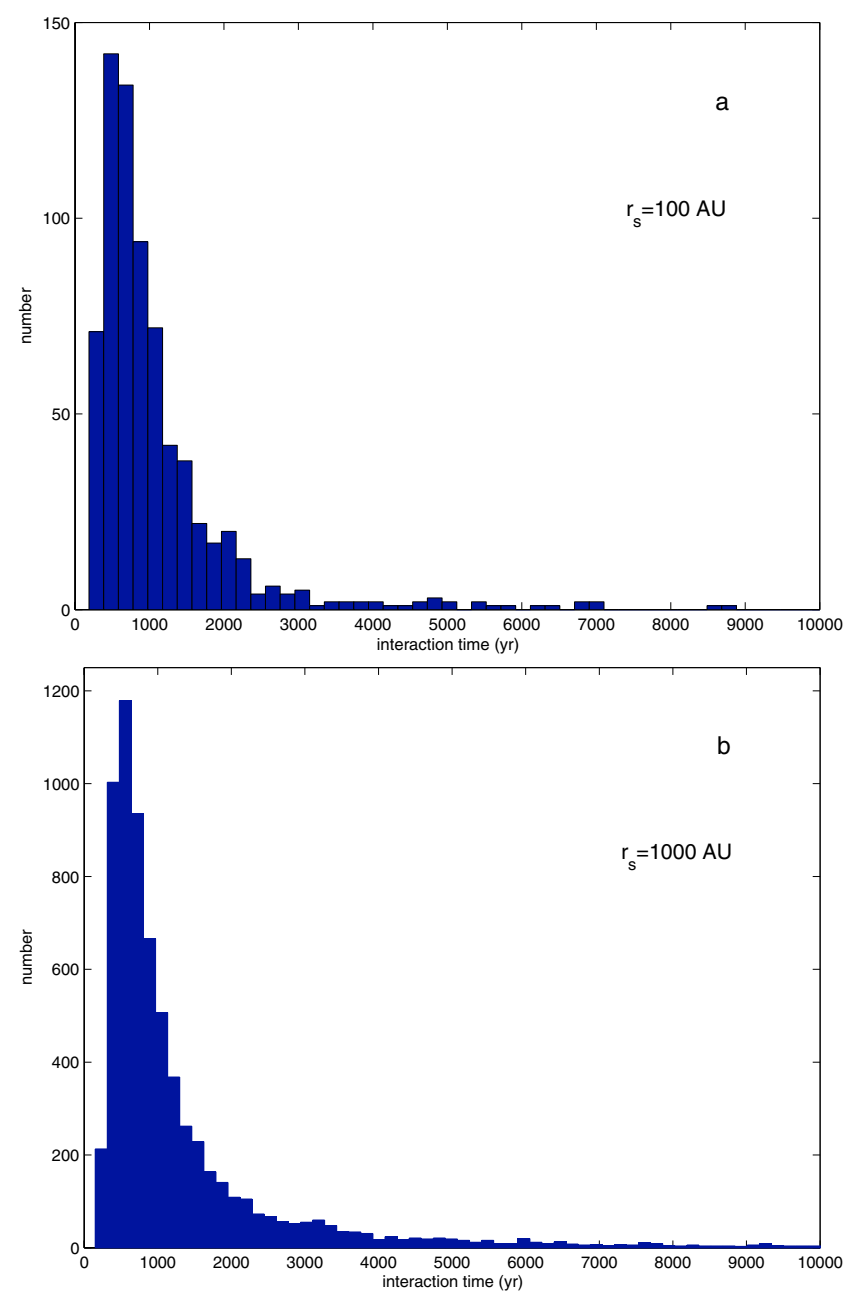

Fig. 2. Interaction time distributions for impulses larger than $0.1 v_{\text {ref }}$. a) Object situated at $r_{\mathrm{s}}=100 \mathrm{AU}$; b) object situated at $r_{\mathrm{s}}=1000 \mathrm{AU}$. In both cases the tail extends beyond $T_{*}=10000 \mathrm{yr}$.

chosen as $v_{\text {ref }}=2 \pi / \sqrt{30000}=0.0363 \mathrm{AU} / \mathrm{yr}$, i.e., the circular speed at $r=3 \times 10^{4}$ AU. For comparison, the aphelion velocity of Sedna for $Q=1000 \mathrm{AU}$ is $0.0742 \mathrm{AU} / \mathrm{yr}$, and if the perihelion were displaced to $35 \mathrm{AU}$ it would be $0.0517 \mathrm{AU} / \mathrm{yr}$. For both the smaller sample size with $r_{\mathrm{s}}=100 \mathrm{AU}$ and the larger one with $r_{\mathrm{s}}=1000 \mathrm{AU}$, the vast majority of the encounters have $T_{*} \lesssim 2000 \mathrm{yr}$ and only a very small fraction have $T_{*}>5000 \mathrm{yr}$. The latter is close to half the orbital period of Sedna and may be taken as the interaction time where the impulse approximation breaks down.
Table 2. Numbers of big impulses $\left(n_{\text {big }}\right)$ and of those that take an interaction time larger than $5000 \mathrm{yr}\left(n_{\text {slow }}\right)$. The ratio of the latter to the former is $f_{\text {slow }}$. The impulse limit $\mathcal{I}_{\text {lim }}$ denotes the ratio $|\Delta v|_{\text {min }} / v_{\text {ref }}$. The experiment in each case involves $1 \times 10^{7}$ random encounters.

\begin{tabular}{r|rrr}
\hline \hline \multicolumn{4}{c}{ a. $\mathcal{I}_{\text {lim }}=1.0$} \\
\hline$r_{\mathrm{s}}(\mathrm{AU})$ & $n_{\text {big }}$ & $n_{\text {slow }}$ & $f_{\text {slow }}$ \\
\hline 100 & 72 & 1 & $1.4 \%$ \\
200 & 122 & 1 & $0.8 \%$ \\
500 & 275 & 10 & $3.6 \%$ \\
1000 & 400 & 17 & $4.3 \%$ \\
\hline \multicolumn{4}{|c}{ b. $\mathcal{I}_{\text {lim }}=0.1$} \\
\hline$r_{\mathrm{s}}(\mathrm{AU})$ & \multicolumn{1}{|c}{$n_{\text {big }}$} & $n_{\text {slow }}$ & $f_{\text {slow }}$ \\
\hline 100 & 718 & 17 & $2.4 \%$ \\
200 & 1454 & 29 & $2.0 \%$ \\
500 & 3367 & 132 & $3.9 \%$ \\
1000 & 6952 & 426 & $6.1 \%$ \\
\hline
\end{tabular}

From the values listed in Table 2 we see that the fraction of the encounters that are too slow does not reach beyond about $6 \%$ even for $|\Delta v|_{\min }=0.1 v_{\text {ref }}$ and $r_{\mathrm{s}}=1000 \mathrm{AU}$ (Fig. 2b), and is smaller for larger $|\Delta \boldsymbol{v}|_{\min }$ or smaller $r_{\mathrm{s}}$. We deem that this is good enough and that the use of $|\Delta v|_{\text {min }}=0.1 v_{\text {ref }}$ is thus warranted, but we would hesitate to use smaller values of $|\Delta v|_{\min }$.

In order to estimate the size of a typical perturbation $|\Delta q|$ caused by $|\Delta \boldsymbol{v}|=|\Delta \boldsymbol{v}|_{\text {min }}=0.1 v_{\text {ref }}$, we take $\left|\Delta v_{t}\right|=|\Delta \boldsymbol{v}|_{\text {min }} / 3$ and use: $\Delta q \simeq 2 q \Delta v_{t} / v_{t}$, where $r v_{t}=h \simeq 52 \mathrm{AU}^{2} \mathrm{yr}^{-1}$ for an orbit with $q=35 \mathrm{AU}$ and $Q=1000 \mathrm{AU}$. We get a $|\Delta q|$ that increases from $0.16 \mathrm{AU}$ at $r=100 \mathrm{AU}$ to $1.6 \mathrm{AU}$ at $r=1000 \mathrm{AU}$. This means that neglecting smaller impulses may imply the neglect of an important small-scale scatter of $q$. We will return to this point below.

In Fig. 3 we show scatter diagrams of $v_{*}$ vs. $d_{\odot}$ for the samples of significant impulses at $r_{\mathrm{s}}=100$ and $1000 \mathrm{AU}$. As explained below, these samples contain many more stellar encounters than we can expect over the age of the Solar System, for the simple reason that we need good statistics. Note the wide range of encounter velocities that contribute in both cases, and the presence of an extended tail of the distribution of minimum distances for $r_{\mathrm{s}}=1000 \mathrm{AU}$ (Fig. 3b). These are due to the inclusion of a small fraction of massive stars into our material (mainly B stars and giants, see Table 1). However, the entries peak around $v_{*} \simeq 25 \mathrm{~km} \mathrm{~s}^{-1}$ and $d_{\odot} \simeq 1000 \mathrm{AU}$ for $r_{\mathrm{s}}=1000 \mathrm{AU}\left(d_{\odot} \simeq 200 \mathrm{AU}\right.$ for $\left.r_{\mathrm{s}}=100 \mathrm{AU}\right)$. Note that a $1 M_{\odot}$ star that passes the Sun or the object at a distance of $1000 \mathrm{AU}$ with a speed of $30 \mathrm{~km} \mathrm{~s}^{-1} \simeq 2 \pi \mathrm{AU} / \mathrm{yr}$ imparts an impulse of $0.006 \mathrm{AU} / \mathrm{yr} \simeq 0.2 v_{\text {ref }}$, while much more distant encounters will yield a significant impulse only if they are slow and the stars are massive. But for $r_{\mathrm{s}}=100 \mathrm{AU}$ the impulses to the object and the Sun from a $d_{\odot} \simeq 1000 \mathrm{AU}$ encounter will largely cancel out, so closer encounters are needed for significant net impulses to result.

We need to emphasize that the samples of $10^{7}$ encounters are much larger than the number of stellar passages within $40000 \mathrm{AU}$ expected during $4 \mathrm{Gyr}$. The latter is close to 1600 , so the ratio is about 6250 . Thus our samples correspond to a time interval of $6250 \times 4 \mathrm{Gyr}=2.5 \times 10^{13} \mathrm{yr}$. In particular, for an object spending $64.5 \%$ of $4 \mathrm{Gyr}$ in the range of 

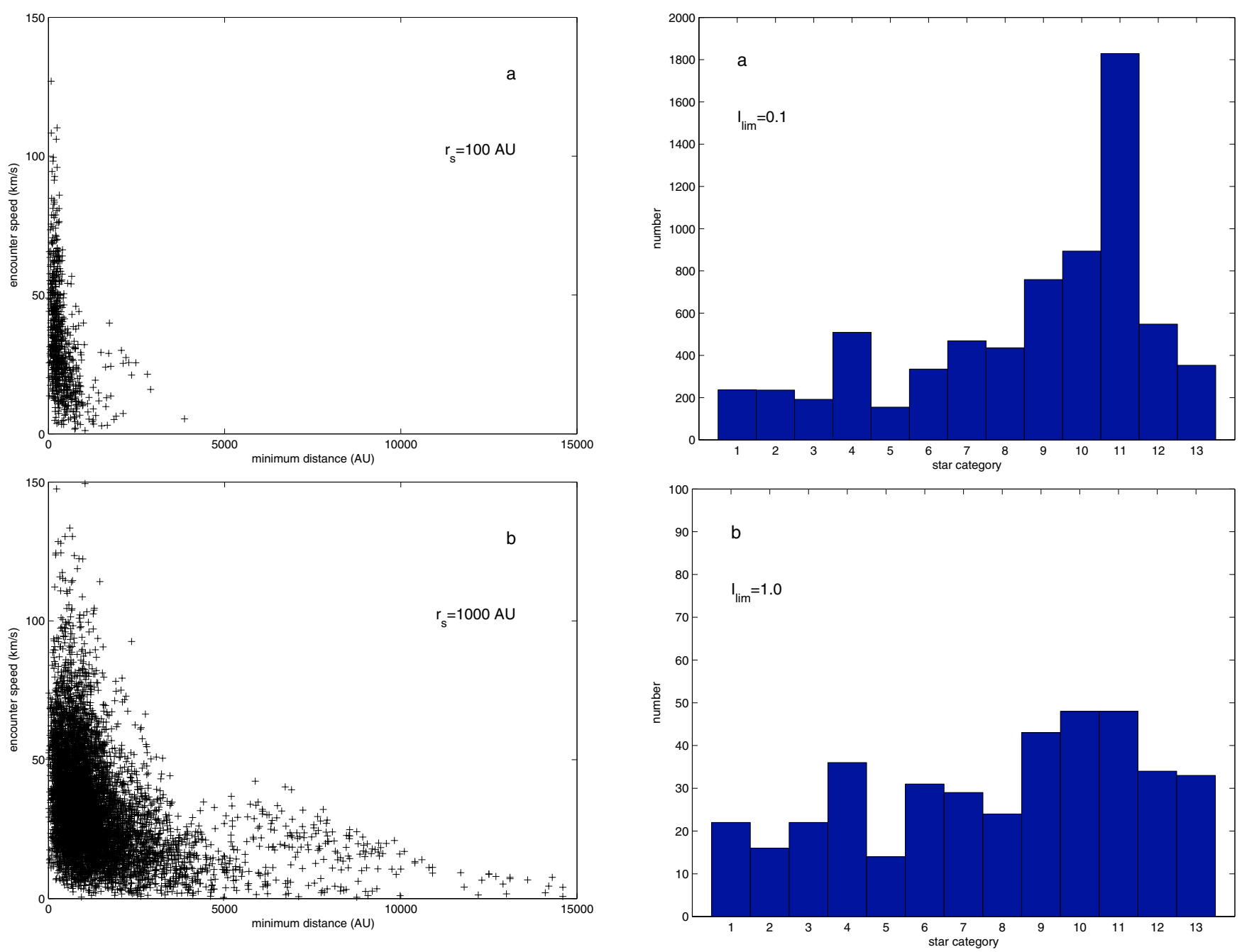

Fig. 3. Encounter speed vs. minimum distance for impulses larger than $0.1 v_{\text {ref }}$. a) 718 entries for an object situated at $r_{\mathrm{s}}=100 \mathrm{AU}$; b) 6952 entries for an object situated at $r_{\mathrm{s}}=1000 \mathrm{AU}$.

$r_{\mathrm{s}}=1000$ AU we expect $0.645 \times 1600 \simeq 1030$ stellar encounters within $40000 \mathrm{AU}$, and for a sample size of 6952 in Fig. 3b (see Table 2b) we should hence expect $1030 \times 6952 / 10^{7} \simeq 0.72$ significant impulses to occur. The contribution from the range of $r_{\mathrm{s}}=500 \mathrm{AU}$ is similarly: $0.262 \times 1600 \times 3367 / 10^{7} \simeq 0.14$, while the contributions from $r_{\mathrm{s}}=100$ and $200 \mathrm{AU}$ are much smaller. The expectation is thus a little less than one significant impulse per object during 4 Gyr.

Figure $4 \mathrm{a}$ shows how the significant impulses are distributed over the 13 stellar categories. Comparing with the relative contributions to the encounter frequency in Table 1, we note that - as expected - the massive star types are overrepresented in the statistics of big impulses. Even so, $\mathrm{M}$ dwarfs yield the dominant contribution. But if we concentrate on very big impulses, as shown in Fig. 4b, the contributions are actually rather evenly distributed over all categories.

Figure 5 shows how the radial direction cosine of the impulse vector is distributed with respect to the modulus of the vector for the $r_{\mathrm{s}}=1000 \mathrm{AU}$ sample. An isotropic distribution of impulse directions would yield a uniform distribution of $C_{r}$. We note that this is nearly the case for all ranges of $|\Delta v|$, even

Fig. 4. Distribution of stellar categories (cf. Table 1) for large impulses suffered by an object at $r_{\mathrm{s}}=1000 \mathrm{AU}$ : a) larger than $0.1 v_{\text {ref }}$; b) larger than $1.0 v_{\text {ref }}$.

though there is a slight concentration to intermediate values of $C_{r}$ of both positive and negative sign.

We have performed one principal Monte Carlo simulation involving 10000 test objects whose starting orbits are characterized by: $q_{0}=35 \mathrm{AU} ; Q_{0}=1000 \mathrm{AU}$. During the $4 \mathrm{Gyr}$ covered by the simulation, 4148 objects received no significant impulse at all - though, as we have seen above, the smaller impulses that we neglected could amount to perturbations of several AU in $q$. The majority of the rest (almost 3000) suffered only one significant impulse, while close to 1000 suffered two, about 300 suffered three and less than 50 suffered four or five. These numbers show clearly that the large perturbations experienced by our test objects mainly result from one close stellar encounter, not a random walk of smaller steps.

A total of 28 objects, i.e., $0.28 \%$ of the sample, were ejected on hyperbolic orbits during a very close stellar encounter. A large fraction of the objects had their perihelion distances decreased by significant amounts, and we have deemed that an orbit with $q<31 \mathrm{AU}$ is unstable against planetary perturbations so that the object will become ejected rapidly from the Solar System. The number of objects thus excluded was 1510 . 


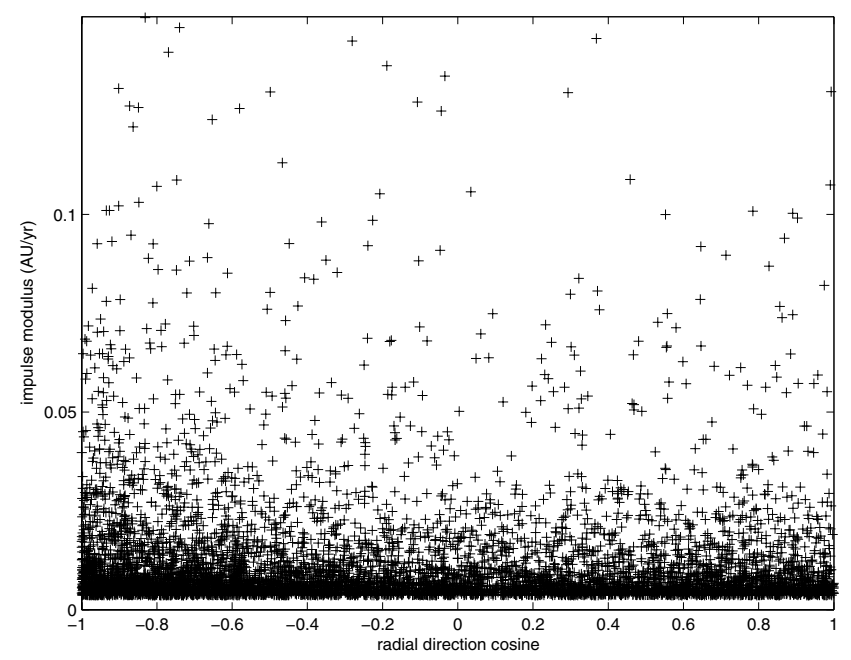

Fig. 5. Impulse modulus vs. radial direction cosine, limited to impulses larger than $0.1 v_{\text {ref }}$, for an object at $r_{\mathrm{s}}=1000 \mathrm{AU}$.

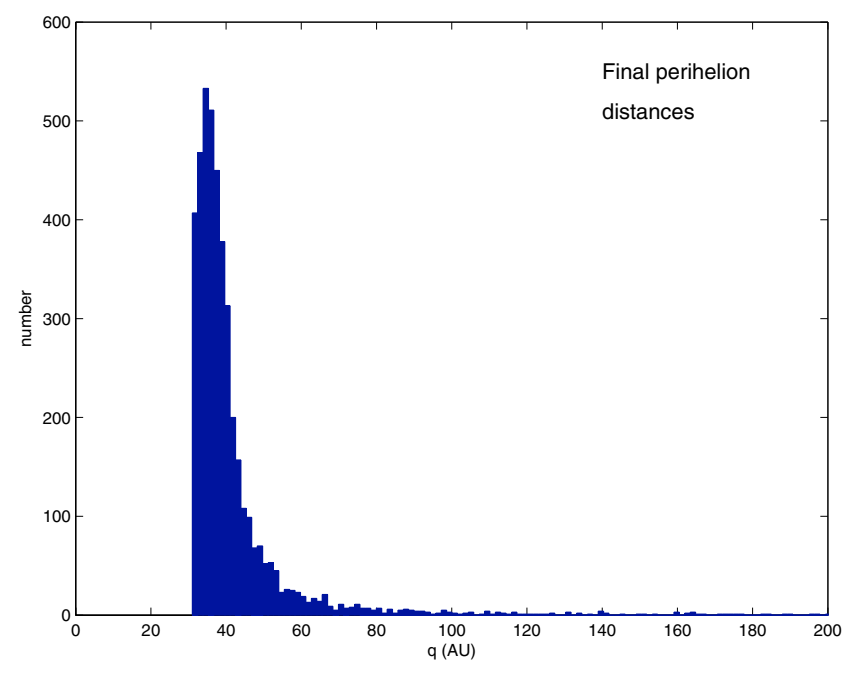

Fig. 6. Distribution of perihelion distances acquired by objects that received significant impulses in a 4 Gyr simulation but stayed at $q>$ $31 \mathrm{AU}$.

Figure 6 shows a histogram of final $q$ values for the 4314 objects that left our principal simulation having suffered significant perturbations. The cut of the abscissa at $q=$ $200 \mathrm{AU}$ is artificial, and the tail in fact extends to $800 \mathrm{AU}$. Interpretations and implications of this diagram will be presented in the next section. Figure 7 shows the result of an additional simulation, including the orbits that were perturbed to $q<31$ AU. We note that a significant percentage of the test objects entered the Saturn-Uranus region and some were even injected directly into Jupiter-crossing orbits.

Given that the far tails of the $q$-distribution in Fig. 7 are expected to originate in one close stellar encounter, we computed the response of $x$-, $y$ - and $z$-disks with 10000 test objects to an encounter with a $1 M_{\odot}$ star moving at $30 \mathrm{~km} \mathrm{~s}^{-1}$. The minimum approach distance was $800 \mathrm{AU}$. In each case the disk had orbital inclinations below $30^{\circ}$ and perihelion distances from 32 to 36 AU. Figure 8 shows the resulting distribution of $a$ and $q$ for $x$-, $y$ - and $z$-disks with initial semimajor axes between

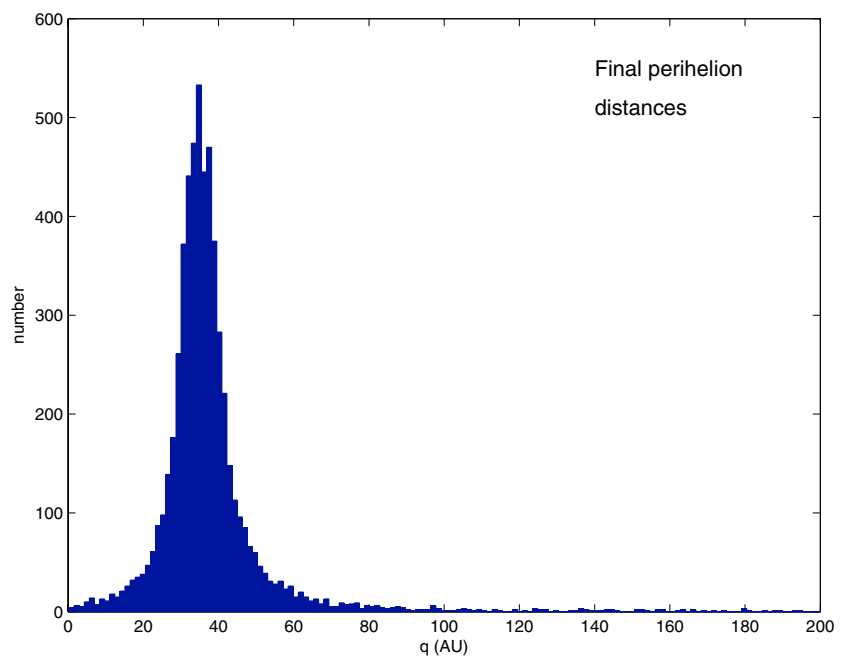

Fig. 7. Distribution of perihelion distances for all objects that received significant impulses in a $4 \mathrm{Gyr}$ simulation, independent of how $q$ evolved.

100 and 600 AU. Note the appearance of a wide scatter in $q$ that starts at $a \sim 300-400$ AU for the $y$-disk and somewhat closer in for the $z$-disk, while the $x$-disk remains much less perturbed over the entire range of $a$. The reason is that the $y$ - and $z$-disks are penetrated by the star, while the $x$-disk is situated on average $800 \mathrm{AU}$ from the stellar track. Thus close encounters between the star and disk objects occur in the former cases but not in the latter.

We also made computations for disks confined to initial $a$ between 400 and $600 \mathrm{AU}$ to get better statistics of the resulting $q$ distribution for Sedna-type orbits. The results are shown in Fig. 9. We see that, while an encounter with the $x$-disk orientation is unable to produce a distribution like that in Fig. 7, both the $y$ - and $z$-disk encounters give results that bear a qualitative resemblance to the result of our long-term Monte Carlo simulation.

\section{Discussion}

Thus, from Figs. 8 and 9 we find a useful confirmation of the simulation, in the sense that an explicit stellar encounter that matches the expectations for the closest ones that ever occurred may indeed reproduce the same scatter of perihelion distances that the simulation yielded. But we also note that different encounter geometries - just like different stellar masses, velocities and distances - yield results that differ to some extent. Therefore we cannot claim to know what the $q$ distribution of the extended scattered disk looks like in detail, but for want of the required detailed knowledge, we can take the simulation results in Figs. 6 and 7 as the best current estimate.

To discuss this further, Fig. 6 should be interpreted as a probability density distribution for the results of stellar perturbations over any $4 \mathrm{Gyr}$ period during the $2.5 \times 10^{13} \mathrm{yr}$ that our impulse samples cover. It is not immediately obvious that this represents the true history of the Solar System, however. In particular, even though the closest encounter distance statistically expected should be a little less than 1000 AU from the Sun, we 

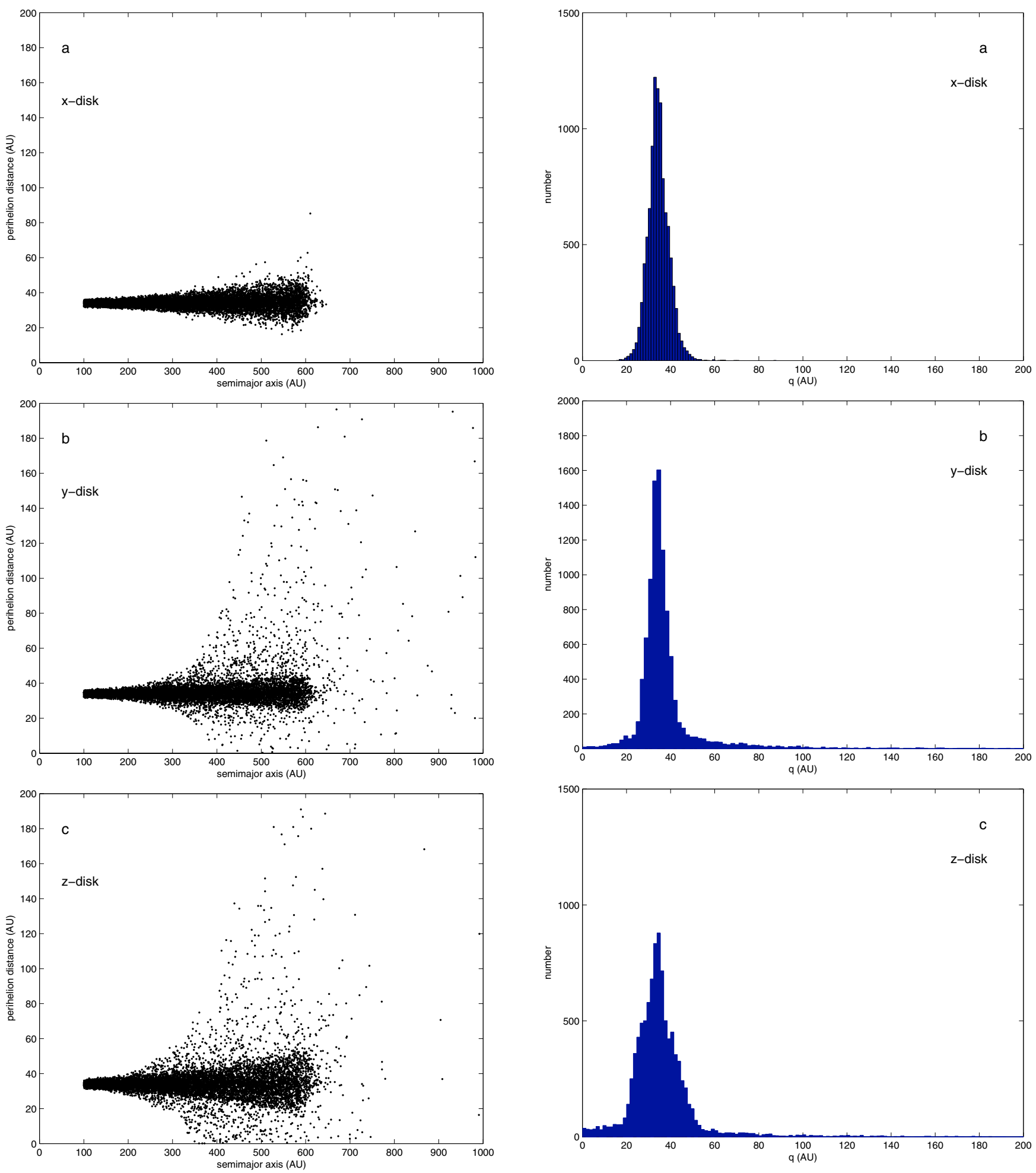

Fig. 8. Semimajor axes and perihelion distances acquired at a stellar passage with $d_{\odot}=800 \mathrm{AU}$ by 10000 objects forming a disk with $q_{0}$ from 32 to $36 \mathrm{AU}$ and $a_{0}$ from 100 to $600 \mathrm{AU}$, for three different orientations of the disk symmetry axis: a) $x$-disk; b) $y$-disk; c) $z$-disk.

cannot be sure that such an encounter has actually occurred. If the closest real encounter was at, e.g., $1500 \mathrm{AU}$ (this would not be too unlikely, if the present encounter frequency is larger than the average in the past), then the whole tail of large

Fig. 9. Perihelion distances acquired through the same stellar passage as in Fig. 8 by 10000 objects forming a disk with $a_{0}$ between 400 and $600 \mathrm{AU}$, for the same disk axis orientations as in Fig. 8.

$q$ values including the orbit of Sedna would likely be absent. On the other hand, the present encounter frequency might also be smaller than it used to be in the past, so that even closer passages could have occurred. In fact, all encounter distances are possible a priori, but Morbidelli \& Levison (2004) did indicate a likely minimum value, as we shall discuss below. 
Indeed, we can only make statements about probabilities, and it is evident that, based on the belief that the current encounter frequency is not an overestimate, an encounter to within $1000 \mathrm{AU}$ or less is not at all unlikely. And when it happened, as verified by Figs. 8b,c and 9b,c, a hypothetical population of proto-ETNOs might have been affected according to a distribution similar to that in Fig. 7, given that the objects would have had random positions at $r \lessgtr 1000 \mathrm{AU}$.

Now, can the existence of one roughly Pluto-sized object in the Sedna orbit be used to infer the existence of a much larger population of such large objects with a $q$ distribution like that in Fig. 6? A tentative answer to this question can be obtained by asserting that, currently, there is no such object at $r<50 \mathrm{AU}$ (otherwise it would be bright enough to have been detected long ago) and only one (i.e., Sedna) at $50<r<100$ AU. The former seems a safer assumption than the latter, and we shall return to this point below. We have computed the fraction of the time spent at $50<r<100$ AU by each object contributing to Fig. 6 plus the 4148 ones remaining in the initial orbit, and formed the sum of all these fractions. This turned out to be about 106, so according to our second assertion we have to scale down our simulated population by a factor 106 . As a result, the number of objects expected to be at $r<50 \mathrm{AU}$ is only about $1 / 2$.

The result of this exercise is a population of 80 objects, of which 1 has $q>90 \mathrm{AU}, 2$ have $60<q<90 \mathrm{AU}, 11$ have $40<$ $q<60 \mathrm{AU}$, and 66 have $q<40 \mathrm{AU}$. Each object except the first has a roughly equal chance of being the one that currently has $r<100 \mathrm{AU}$, so it does not appear too unlikely for Sedna to be the one. However, from Fig. 8 we see that the picture just described cannot be complete. If the very large population with $q \lesssim 35$ AU has survived until the present time, so should also the fraction of the initial scattered disk with $a \lesssim 200 \mathrm{AU}$, whose perihelion distances have remained small. If that were the case, we should have another, even larger population of large objects with $q \lessgtr 35 \mathrm{AU}$ and $a \lesssim 200 \mathrm{AU}$, and it would be unbelievable that none have been observed.

Obviously, our discussion has missed two unmodelled sources of perturbations of $q$ : the field of more distant, or in any case less efficient, stellar encounters, and the gravitational influence of the planets. As to the first one, we have seen that the effects may amount to a cumulative $|\Delta q| \gtrsim 1 \mathrm{AU}$. Gladman et al. (2002) found that interactions with Neptune may raise $q$ to $36 \mathrm{AU}$, and thus the inverse is also possible. The combination of the two mechanisms may yield an efficient erosion of the scattered disk so that, effectively, no Sedna-sized objects remain today with $q \lesssim 36 \mathrm{AU}$.

We are thus led to conjecture that the decisive stellar encounter occurred early on, before the erosion had time to occur and the scattered disk may have been very massive. Morbidelli \& Levison (2004) argue that the encounter must have occurred before the Oort cloud was created - and thus at an early epoch - because otherwise the cloud would have been disrupted. We note that this is natural in the cluster scenario for which Morbidelli and Levison's simulation is relevant, because on the one hand the stellar encounter is expected to be very slow and thus able to disrupt the Oort cloud, and on the other hand it must have occurred very early, before the cluster was disrupted. Hence there should have been time to rebuild the Oort cloud afterwards.

But even so, we believe that an early stellar encounter is necessary, since if it occurred too late, it would have been impossible to create the above number of objects the size of Sedna in the extended scattered disk. The current mass of the extended scattered disk is very uncertain, being dependent both on the actual number of Sedna-sized objects and the slope of the size distribution (Morbidelli, priv. comm.). The mass of the primordial scattered disk is uncertain too, as is the "erosional" half life against ejection by Neptune. Further research is needed in order to say exactly how early an epoch is required, and whether a connection to a stellar cluster where the Sun was born (Brown et al. 2004) is indeed likely. If the scattered disk was strongly perturbed by an encounter with a cluster member, and if that is the main reason that the ETNO population exists, we still expect our results from encounters with random field stars to be valid for the remaining part of the disk until the present time.

In any case, with the scenario proposed we may assume that essentially the whole population with $q<40$ AU in Fig. 6 has been stripped away, and only the part with $q>40$ AU exists today. Repeating thus the above analysis to find the size of such an ETNO population on the assumption that only one or two currently have $50<r<100 \mathrm{AU}$, we get $\sim 5-10$ objects with $q>90 \mathrm{AU}, \sim 10-20$ objects with $60<q<90 \mathrm{AU}$, and $\sim 50$ 100 objects with $40<q<60 \mathrm{AU}$. In fact there is no special justification for that assumption. Judging from the sky coverage of present surveys, Brown et al. (2004) estimate a likely number of $\sim 6$, for which case the quoted numbers will have to be scaled upward by a factor of $\sim 4$.

To summarize, we find that our simulation has borne out Morbidelli and Levison's suggestion of an early, close stellar encounter as a likely mechanism to explain the Sedna orbit. In addition, we are led to expect the existence of a large number of similar-sized or larger objects with perihelion distances from $\sim 40 \mathrm{AU}$ outward. If the total number is $\lesssim 80$, it is likely that Sedna is currently alone in the $50<r<100$ AU range. More likely, the total number is several hundred, and Sedna has several as yet undiscovered colleagues of comparable brightness in the same range of $r$. A comment on the circumstance that the first discovered object belongs to a minority of $\sim 10 \%$ with very large $q$ is in order. We do not believe this minority to be so small as to call for a special explanation. Moreover, even though a stellar encounter distance less than $800 \mathrm{AU}$ might have stirred up the scattered disk more efficiently, we noted in the Introduction that Morbidelli \& Levison (2004) give a strong argument against such a scenario in that the inner part of the scattered disk would also have been excited and the nondiscovery of Sedna-like objects with much smaller semimajor axes would thus be difficult to explain.

It is tempting to speculate that one or a few objects with $q \sim 50-55 \mathrm{AU}$, where a large part of the population should be, may even be much larger than Sedna, and may thus offer an alternative to the "rogue planet" scenario for explaining the apparent edge of the Kuiper belt at $\sim 50 \mathrm{AU}$ (Brunini \& Melita 2002). Each such object might have made hundreds of thousands of perihelion passages since they were emplaced. We find it very interesting to investigate the possible sculpting effect of 
those passages on a population in the 50-60 AU range, as well as the "heating" effect on the orbital distribution at somewhat smaller distances.

Let us now discuss the implications of Figs. 7 and 9. If indeed the scattered disk was massive enough for the close stellar encounter to have transferred hundreds of objects the size of Sedna or larger with $a \gtrsim 300$ AU into large values of $q$, then a large number of such objects would also have been injected into planet-crossing orbits. For any reasonable size distribution, the number of objects with diameters of $\sim 100 \mathrm{~km}$ or smaller would have been enormous. Thus a huge population of large objects would have invaded the inner Solar System. One may wonder whether this is a possible scenario for explaining the late heavy bombardment of the terrestrial planets and the Moon (Hartmann et al. 2000), along the lines discussed by Levison et al. (2001). However, the stellar encounter that created the ETNO population must likely have occurred much earlier. A more attractive scenario might be that this encounter happened very early (possibly within a stellar cluster) and that later on a random field star passed very close about 4 Gyr ago with relatively little effect on the ETNO population but injecting an important gust of objects into the inner Solar System.

\section{Conclusions}

1. Based on the current stellar encounter frequency and distribution of stellar masses and velocities, statistically for an arbitrary interval of $4 \mathrm{Gyr}$, an object with perihelion distance $q_{0}=35 \mathrm{AU}$ and aphelion distance $Q_{0}=1000 \mathrm{AU}$ is found to stand a $40 \%$ chance of receiving only slight stellar impulses that cause perturbations less than a few $\mathrm{AU}$ in $q$, a $15 \%$ chance of being brought by larger impulses into $q<31$ AU and thereby likely being ejected from the Solar System, and a $45 \%$ chance of receiving significant stellar impulses that keep $q>31 \mathrm{AU}$ and lead to a wide distribution of $q$, extending nearly all the way to $1000 \mathrm{AU}$.

2. For the latter category, a population with a large scatter of $q$ would likely arise mainly from one close stellar encounter, and our picture is consistent with, e.g., a $1 M_{\odot}$ star passing at $30 \mathrm{~km} \mathrm{~s}^{-1}$ with a minimum distance of $800 \mathrm{AU}$, thereby exciting a pre-existing scattered disk with perihelia somewhat outside Neptune's orbit and extending to $\sim 1000 \mathrm{AU}$.

3. The existence of minor planet (90377) Sedna in its present orbit is consistent with such a scenario, but in order to offer a viable explanation the decisive stellar encounter must have occurred early enough that the scattered disk was still rich and massive. Detailed constraints on the timing of that encounter will depend on estimates of the mass of the ETNO population and of the "erosional" half-life of the scattered disk against close encounters with Neptune and ejection from the Solar System.
4. If the picture outlined is correct and the massive scattered disk did exist, then since no Sedna-sized objects have been observed with $q \simeq 35 \mathrm{AU}$ and $a<200 \mathrm{AU}$, it is clear that the "inner" part of the scattered disk must have been nearly fully eroded away. This means that an "outer" part with $a \simeq 500$ AU must have been heavily eroded too. An early epoch of the decisive stellar encounter is thus favoured, and moreover it is possible to extrapolate the current presence of Sedna in the $50<r<100$ AU range into a total population of $\gtrsim 100$ objects of the same size or larger in stable orbits with $q \gtrsim 40 \mathrm{AU}$ and $a \gtrsim 300$ AU.

5. Tentatively, such a population may contain objects large enough to have sculpted the outer edge of the Kuiper belt, but which are currently too far away to have been detected. If the picture of an early, decisive stellar encounter is correct, then certainly it must have been followed by a surge in the flux of large, near-Earth objects. Whether such an encounter may be related to the late heavy bombardment may warrant further investigation.

Acknowledgements. HR gratefully acknowledges the hospitality of the Observatoire de Nice during a stay there as visiting scientist in Nov.-Dec. 2003, when the basic work on computer codes used in this paper was done. Research grants from the Swedish National Space Research Board and the Swedish Council of Science were also very beneficial for this work.

\section{References}

Allen, C. W. 1985, Astrophysical Quantities, 3rd ed. (London: Athlone Press)

Brown, M. E., Trujillo, C., \& Rabinowitz, D. 2004, ApJL, submitted Brunini, A., \& Melita, M. 2002, Icarus, 160, 32

Dones, L., Weissman, P. R., Levison, H. F., \& Duncan, M. J. 2004, in Comets II, ed. M. C. Festou, et al. (Tucson: Univ. Arizona), in press

Fernández, J. A. 1997, Icarus, 129, 106

Fernández, J. A., \& Brunini, A. 2000, Icarus, 145, 580

García-Sánchez, J., Weissman, P. R., Preston, R. A., et al. 2001, A\&A, 379,634

Gladman, B., Holman, M., Grav, T., et al. 2002, Icarus, 157, 269

Gomes, R. S. 2003, Earth, Moon \& Planets, 92, 42

Hartmann, W. K., Ryder, G., Dones, L., \& Grinspoon, D. 2000, in Origin of the Earth and Moon, ed. R. M. Canup, \& K. Righter (Tucson: Univ. Arizona), 805

Levison, H. F., Dones, L., Chapman, C. R., et al. 2001, Icarus, 151, 286

Marsden, B. G. 2004, Minor Planet Electronic Circular 2004-E45

Morbidelli, A., \& Brown, M. E. 2004, in Comets II, ed. M. C. Festou et al. (Tucson: Univ. Arizona), in press

Morbidelli, A., \& Levison, H. F. 2004, AJ, submitted 